\title{
Toxoplasmosis: a rare cause of IRIS in HIV infected patients. Case series
}

\author{
Ruxandra Moroti ${ }^{1,2^{*}}$, Daniela Munteanu', Mihaela Rădulescu ${ }^{1,2}$, Adriana Hristea ${ }^{1,2}$, lulia Niculescu ${ }^{1,2}$, \\ Raluca Mihăilescu', Roxana Petre ${ }^{1,3}$, Raluca Hrişcă1,3, Raluca Jipa ${ }^{1,3}$, Ana Maria Petrescu', Maria Nica ${ }^{4}$, Mihai Lazăr ${ }^{1,2}$, \\ Anca-Ruxandra Negru', Irina Lăpădat ${ }^{1}$, Angelica Teniță' , Victoria Aramă'1,2 \\ From The 9th Edition of the Scientific Days of the National Institute for Infectious Diseases Prof Dr Matei Bals \\ Bucharest, Romania. 23-25 October 2013
}

\section{Background}

Cerebral toxoplasmosis is one of the main 3 intracerebral opportunistic infections in HIV positive patients, along with cryptococcosis and tuberculosis. In comparison to these last 2 entities, toxoplasmosis does not provoke or very rarely provokes reconstitution syndromes.

\section{Methods}

We analyzed a case series of 3 patients with cerebral toxoplasmosis admitted in the Adults III Department of the National Institute for Infectious Diseases "Prof. Dr. Matei Balş" in 2012-2013.

\section{Results}

Three patients, one male and 2 women, aged 55 years old, respectively 41 and 42 year-old, all 3 diagnosed concomitantly with HIV infection (as very late presenters) and cerebral toxoplasmosis, with a CD4 count of 6,6 and $7 / \mathrm{cmm}$ respectively, viral loads (VL) of 254,000, 57,000 and 156,000 copies/mL respectively, and CSF viral load below the plasmatic VL in all 3 cases. We recorded minimal abnormalities of CSF analysis regarding the number of cells and biochemical exams; all had positive PCR for Toxoplasma gondii in the CSF and positive serology (IgG). All 3 had intracerebral lesions (abscesses) and all were biopsied at the neurosurgery department for diagnostic purpose before knowing their HIV-positive status. They received high doses of oral trimethoprim/sulfamethoxazole (T/S) for toxoplasmosis and antiretroviral therapy in the first 2 weeks after the diagnosis. They repeated cerebral imagery (MRI) after 3 weeks of T/S and had no regression of the size of lesions (although with the decreasing of perilesional edema) and new lesions, in two cases without having corresponding symptoms; in all 3 cases the CD4 count increased in the first month more than $100 \%$. The search for another cause for the augmentation of their brain lesions was negative. Maintaining the same medication, the next imagery exams showed improvement in 2 out of 3 cases, in which the outcome was favorable with almost complete neurological recovery. In the remaining case the evolution was unfavorable (death).

\section{Conclusions}

In our 3 cases we presumed a paradoxical toxoplasmosis IRIS, with little or no clinical deterioration strictly linked with imagery exams depreciation in 2 out of 3 cases but with a fatal evolution in one case. Even rarely reported, the toxoplasmosis IRIS could be taken into account in some situations.

\begin{abstract}
Authors' details
${ }^{1}$ National Institute for Infectious Diseases "Prof. Dr. Matei Balş", Bucharest, Romania. ${ }^{2}$ Carol Davila University of Medicine and Pharmacy, Bucharest, Romania. ${ }^{3}$ Central Universitary Emergency Military Hospital Dr Carol Davila, Bucharest, Romania. ${ }^{4}$ Clinical Hospital of Infectious and Tropical Diseases "Dr. Victor Babeş", Bucharest, Romania.
\end{abstract}

Published: 16 December 2013

doi:10.1186/1471-2334-13-S1-07

Cite this article as: Moroti et al.: Toxoplasmosis: a rare cause of IRIS in HIV infected patients. Case series. BMC Infectious Diseases 2013 13(Suppl 1):07.

\footnotetext{
* Correspondence: ruxandra_moroti@yahoo.com

'National Institute for Infectious Diseases "Prof. Dr. Matei Balş", Bucharest, Romania

Full list of author information is available at the end of the article
}

(c) 2013 Moroti et al; licensee BioMed Central Ltd. This is an Open Access article distributed under the terms of the Creative Commons 